\title{
Inconsistency evaluation in pairwise comparison using norm-based distances
}

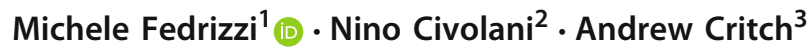

Received: 14 December 2019 / Accepted: 11 August 2020 / Published online: 30 August 2020

(c) The Author(s) 2020

\begin{abstract}
This paper studies the properties of an inconsistency index of a pairwise comparison matrix under the assumption that the index is defined as a norm-induced distance from the nearest consistent matrix. Under additive representation of preferences, it is proved that an inconsistency index defined in this way is a seminorm in the linear space of skew-symmetric matrices and several relevant properties hold. In particular, this linear space can be partitioned into equivalence classes, where each class is an affine subspace and all the matrices in the same class share a common value of the inconsistency index. The paper extends in a more general framework some results due, respectively, to Crawford and to Barzilai. It is also proved that norm-based inconsistency indices satisfy a set of six characterizing properties previously introduced, as well as an upper bound property for group preference aggregation.
\end{abstract}

Keywords Inconsistency index · Pairwise comparison matrix · Norm · Distance

JEL Classification C44 · D7

\section{Introduction}

Pairwise comparison over a set of alternatives $X=\left\{x_{1}, \ldots, x_{n}\right\}$ is a well known and powerful method for preference elicitation in a decision problem. An important characteristic of this method is the capability of dealing with the imprecision of the collected data due to the unavoidable inconsistency of human judgements. Each entry $a_{i j}$ of a pairwise comparison matrix, $P C M$ in the following, $\mathbf{A}=\left(a_{i j}\right)_{n \times n}$ quantifies

$\bowtie \quad$ Michele Fedrizzi

michele.fedrizzi@unitn.it

1 Department of Industrial Engineering, University of Trento, Via Sommarive 77, 38123 Trento, Italy

2 University of Trento, Trento, Italy

3 Center for Human-Compatible AI, University of California, Berkeley, California, USA 
the degree of preference of alternative $x_{i}$ over alternative $x_{j}$. Two widely used representations of preferences are the so-called multiplicative and additive ones. In the multiplicative approach (Saaty 1977), $a_{i j}$ is the relative preference of alternative $x_{i}$ over alternative $x_{j}$, and therefore, it estimates the ratio between the weight $w_{i}$ of $x_{i}$ and the weight $w_{j}$ of $x_{j}, a_{i j} \approx \frac{w_{i}}{w_{j}}$ Conversely, in the additive approach, $a_{i j}$ estimates the difference between the weights of $x_{i}$ and $x_{j}$, respectively, $a_{i j} \approx w_{i}-w_{j}$. Therefore, different assumptions in preference quantification correspond to different meaning of the entries $a_{i j}$. It has been proved that the multiplicative and the additive representations are isomorphic and, therefore, equivalent. In fact, a multiplicative $P C M$ $\left(a_{i j}\right)_{n \times n}$ can be easily transformed into a corresponding additive $P C M$ by componentwise applying the logarithmic function, thus obtaining $\left(\ln \left(a_{i j}\right)\right)_{n \times n}$. Details on this isomorphism can be found in (Barzilai 1998; Cavallo and D'Apuzzo 2009). In the multiplicative approach, a pairwise comparison matrix PCM is a positive real-valued matrix $\mathbf{A}=\left(a_{i j}\right)_{n \times n}$ with $a_{i i}=1 \forall i$ and $a_{i j} a_{j i}=1 \forall i, j$. Multiplicative reciprocity $a_{i j} a_{j i}=1$ derives from $\frac{w_{i}}{w_{j}} \frac{w_{j}}{w_{i}}=1$, and this property is always required. A PCM is said consistent if and only if

$$
a_{i j} a_{j k}=a_{i k}, \quad i, j, k=1, \ldots, n .
$$

Consistency condition (1) corresponds to the ideal situation where the decision maker is perfectly coherent in her/his judgements and it is in general not required, since it is well known that in making paired comparisons people do not have the intrinsic logical ability to always be consistent (Saaty 1994). Despite a violation of (1) to some extent is necessarily accepted, consistency has always been regarded as a desirable property, since coherent judgements are clearly considered more preferable than contradictory ones. Therefore, a correct inconsistency evaluation is regarded as a crucial task and several indices have been proposed in order to quantify the deviation from the condition of full consistency (1).

The problem of inconsistency evaluation has been addressed by means of many different proposals. We briefly cite some of them. Saaty (1977) proposed, in his seminal paper, the first and still most popular inconsistency index. Koczkodaj (1993) and Duszak and Koczkodaj (1994) introduced an inconsistency index which became very popular too. Bozóki and Rapcsák (2008) summarized the relationship between some weighting methods and the corresponding inconsistency indices. Then, the authors compared Saaty's and Koczkodaj's inconsistency indices. Recently, Brunelli and Fedrizzi (2019) proposed a general formulation for inconsistency indices that includes many among the known indices. Cavallo (2020) studied the functional relations between some important consistency indices, mainly focusing on the case $n=3$. Nevertheless, the detailed description of all the relevant contributions on the consistency evaluation is beyond the scope of this paper. For a comprehensive survey, see (Brunelli 2018). Most of these studies assume the multiplicative representation of the preferences, i.e., they deal with multiplicative PCMs. Some papers take into account both the multiplicative and the additive representations (Fichtner 1984; Chu 1998). Brunelli et al. (2013) compared ten known indices numerically, while Brunelli (2017), Brunelli and Fedrizzi (2015a) and Csató (2018, 2019) proposed a more theoretical approach by studying some axioms for inconsistency indices. In Cavallo and D' Apuzzo 
(2009) and in some following papers by the same authors, a general framework for inconsistency evaluation is proposed, based on the algebraic structure of group. Ramík and Korviny (2010) proposed a distance-based evaluation of the inconsistency in the framework of fuzzy sets. Other interesting distance-based approaches to the study of inconsistency have been proposed more recently by Brunelli and Cavallo (2020) and Mazurek and Ramík (2019).

This paper proposes a geometric-oriented unifying point of view for inconsistency evaluation based on norm-induced metrics in matrix spaces. Our proposal can be considered as a generalization of the approach by Crawford and Williams (1985), where the logarithmic least square method (LLSM) is applied. In fact, their method corresponds to the Euclidean norm minimization after passing to the additive representation of preferences. This justifies, in our view, the numerous good properties of the LLSM and the related geometric mean solution.

The rest of the document is organized as follows. After some preliminaries in Sect. 2, we assume the additive representation of preferences, and in Sect. 3, we introduce an inconsistency index for a $P C M$ defined as a norm-induced distance from the nearest consistent $P C M$. More precisely, in Sect. 3.1 we partition the subspace of $P C M \mathrm{~s}$ (skewsymmetric matrices) into equivalence classes, so that each class will correspond to a single inconsistency value. In Sect. 3.2, we define our index and we prove that it is a seminorm in the vector space of $P C M$ s. We prove, respectively, in Sect. 3.3 and in Sect. 3.4, that our index satisfies a set of six axioms previously introduced and an upper bound property for group preference aggregation. Finally, in Sect. 4, we discuss some conclusions and future work.

\section{Preliminaries}

For a fixed $n>2$, let $\mathbb{R}^{n \times n}$ be the vector space of $n$-order real matrices. Let $\mathcal{A} \subset \mathbb{R}^{n \times n}$ be the set of multiplicative $P C M$ s of order $n$,

$$
\mathcal{A}=\left\{\mathbf{A}=\left(a_{i j}\right)_{n \times n} \mid a_{i j}>0, a_{i j} a_{j i}=1 \forall i, j\right\} .
$$

Similarly, the set of consistent multiplicative $P C M \mathrm{~s} \mathcal{A}^{*} \subset \mathcal{A}$ is defined as

$$
\mathcal{A}^{*}=\left\{\mathbf{A}=\left(a_{i j}\right)_{n \times n} \mid \mathbf{A} \in \mathcal{A}, a_{i k}=a_{i j} a_{j k} \forall i, j, k\right\} .
$$

An inconsistency index is a function which associates a real number to each $P C M$ $\mathbf{A} \in \mathcal{A}$,

$$
I: \mathcal{A} \rightarrow \mathbb{R}
$$

The number $I(\mathbf{A})$ quantifies the inconsistency of $\mathbf{A}$.

A PCM $\mathbf{A}=\left(a_{i j}\right)_{n \times n}$ can be viewed as a point in the vector space $\mathbb{R}^{n \times n}$. Closeness to consistency condition (1) can therefore be interpreted as closeness of $\mathbf{A}$ to a consistent matrix $\mathbf{A}_{C}$ in the same space. It is interesting to observe that choosing the 'closest' consistent matrix $\mathbf{A}_{C}$ exactly corresponds to compute the weight vector $\mathbf{w}$ from $\mathbf{A}$. In 
fact, $\mathbf{A}_{C}$ is consistent if and only if there exists a weight vector $\mathbf{w}=\left(w_{1}, \ldots, w_{n}\right)$ such that $\mathbf{A}_{C}=\left(\frac{w_{i}}{w_{j}}\right)$. Every method for deriving a weight vector $\mathbf{w}=\left(w_{1}, \ldots, w_{n}\right)$ from an inconsistent $P C M \mathbf{A}$ identifies a consistent matrix $\mathbf{A}_{C}=\left(\frac{w_{i}}{w_{j}}\right)$ which is considered the 'closest' one to $\mathbf{A}$ according to a certain metric in $\mathbb{R}^{n \times n}$. Then, deriving a weight vector and measuring inconsistency can be integrated into one process by minimizing an appropriate objective function such that its minimum point is the weight vector, while the optimal value of the objective function gives the inconsistency of the pairwise comparison matrix. These observations suggest to characterize an inconsistency index by means of the notion of distance. Nevertheless, it is convenient to first shift to the additive representation of preferences. This can be done by a simple componentwise logarithmic transformation and allows working with the powerful tools of linear algebra. Note that the multiplicative and the additive representation of preferences are isomorphic, as described in detail by Barzilai (1998) and Cavallo and D'Apuzzo (2009).

By componentwise applying a logarithmic function to a $P C M \mathbf{A}=\left(a_{i j}\right)$,

$$
\ln \left(a_{i j}\right)=r_{i j}
$$

a skew-symmetric matrix $\mathbf{R}=\left(r_{i j}\right)$ is obtained, since multiplicative reciprocity $a_{i j} a_{j i}=1$ is transformed into additive reciprocity

$$
r_{i j}+r_{j i}=0, \quad i, j=1, \ldots, n \text {. }
$$

Being (3) a homogenous linear system, the image set of $\mathcal{A}$ through the logarithmic function is the linear subspace of $\mathbb{R}^{n \times n}$ of the skew-symmetric matrices, say $\mathcal{L}=$ $\{\ln (\mathbf{A}) ; \mathbf{A} \in \mathcal{A}\}=\ln (\mathcal{A})$. The dimension of $\mathcal{L}$ is $n(n-1) / 2$, i.e., the number of the upper-diagonal entries.

A consistent matrix $\mathbf{A}=\left(a_{i j}\right) \in \mathcal{A}^{*}$ is transformed into a skew-symmetric matrix $\mathbf{R}=\ln (\mathbf{A})=\left(\ln \left(a_{i j}\right)\right)=\left(r_{i j}\right)$ satisfying

$$
r_{i j}+r_{j k}=r_{i k}, \quad i, j, k=1, \ldots, n .
$$

Property (4) clearly follows from (1) and proves that the image set of $\mathcal{A}^{*}$ through the logarithmic function is a linear subspace of $\mathcal{L}$, and therefore a linear subspace of $\mathbb{R}^{n \times n}$, say $\mathcal{L}^{*}=\left\{\ln (\mathbf{A}) ; \mathbf{A} \in \mathcal{A}^{*}\right\}=\ln \left(\mathcal{A}^{*}\right)$ (Kocakodaj 1997). The dimension of $\mathcal{L}^{*}$ is $n-1$ since it is known that $n-1$ entries are necessary and sufficient to completely identify a consistent $n$-order matrix, provided that they are adequately chosen (Chu 1998; Fedrizzi et al. 2019).

Since $\mathcal{A}$ is isomorphic to $\mathcal{L}$ and linear spaces are simple structures where it is possible working with tools of linear algebra, then, from now on, we will study preference inconsistency in the subspace $\mathcal{L}$. Therefore, we will use the usual notation $\mathbf{A}$ also to denote a skew-symmetric matrix in $\mathcal{L}$. An inconsistency index will be a function

$$
I: \mathcal{L} \rightarrow \mathbb{R}
$$

and we will call 'additive PCM' a matrix in $\mathcal{L}$. 


\section{Distances and norms}

As remarked above, an inconsistency index measures to which extent a preference matrix is far from consistency. Since distance is the most suitable mathematical tool to evaluate how much two objects are different, it is quite natural to define an inconsistency index $I(\mathbf{A})$ of a matrix $\mathbf{A} \in \mathcal{L}$ as the distance of $\mathbf{A}$ from the linear subspace $\mathcal{L}^{*}$ of consistent matrices,

$$
I_{d}(\mathbf{A})=d\left(\mathbf{A}, \mathcal{L}^{*}\right) .
$$

Nevertheless, the notion of distance is too general to conveniently characterize inconsistency, and definition (6) can lead to unsatisfactory inconsistency indices (Fichtner 1986).

In the following, we will prove that the problem can be overcome by restricting metrics to those defined by norms. The resulting inconsistency indices satisfy a relevant set of good properties. Before proceeding, let us recall the basic notions of distance, norm and seminorm.

Definition 1 (Distance) A distance, or metric, on a given set $Z$ is a function $d$ : $Z \times Z \rightarrow \mathbb{R}$ that satisfies the following three conditions,

1. $d(x, y) \geq 0 \quad \forall x, y \in Z$ and $d(x, y)=0 \Leftrightarrow \quad x=y$.

2. $d(x, y)=d(y, x) \quad \forall x, y \in Z \quad$ (symmetry).

3. $d(x, z) \leq d(x, y)+d(y, z) \quad \forall x, y, z \in Z \quad$ (triangle inequality).

Definition 2 (Norm) A norm on a vector space $V$ is a function $\|\cdot\|: V \rightarrow \mathbb{R}$ that satisfies the following conditions,

1. $\|\mathbf{x}\| \geq 0 \quad \forall \mathbf{x} \in V \quad$ (nonnegativity).

2. $\|\lambda \mathbf{x}\|=|\lambda|\|\mathbf{x}\| \quad \forall \mathbf{x} \in V, \quad \forall \lambda \in \mathbb{R}$ (homogeneity).

3. $\|\mathbf{x}+\mathbf{y}\| \leq\|\mathbf{x}\|+\|\mathbf{y}\| \quad \forall \mathbf{x}, \mathbf{y} \in V \quad$ (triangle inequality).

4. $\|\mathbf{x}\|=0 \Leftrightarrow \mathbf{x}=\mathbf{0} \quad$ (strict positivity)

A seminorm on $V$ is a function $s: V \rightarrow \mathbb{R}$ that satisfies the first three conditions above. In other words, a seminorm has the same properties as a norm, except that it may have a zero value for some nonzero vectors.

\subsection{A partition of $\mathcal{L}$ into equivalence classes}

The vector space structure of $\mathcal{L}$ and $\mathcal{L}^{*}$ naturally induces a partition of $\mathcal{L}$ into equivalence classes. Since $\mathcal{L}^{*}$ is a subspace of $\mathcal{L}$, it is possible to consider the quotient space $\mathcal{L} / \mathcal{L}^{*}$. More formally, the quotient space $\mathcal{L} / \mathcal{L}^{*}$ is defined as follows, using the corresponding equivalence relation.

Definition 3 (Quotient space) Consider the equivalence relation in $\mathcal{L}$

$$
\mathbf{A} \sim \mathbf{B} \Leftrightarrow \mathbf{B}-\mathbf{A} \in \mathcal{L}^{*} \quad \mathbf{A}, \mathbf{B} \in \mathcal{L} .
$$




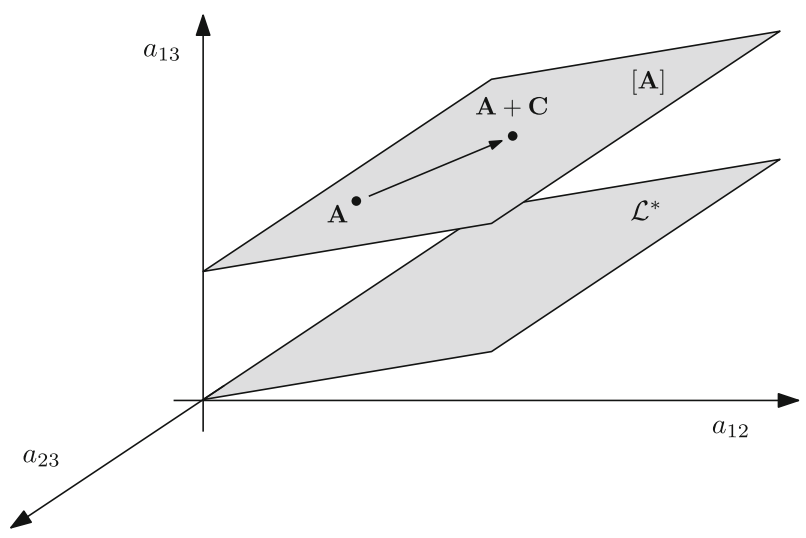

Fig. 1 Example of an equivalence class $[\mathbf{A}]$ for $n=3$

The quotient set $\mathcal{L} / \mathcal{L}^{*}$ is defined as

$$
\mathcal{L} / \mathcal{L}^{*}=\mathcal{L} / \sim
$$

and it is a vector space with the operations naturally induced by the operations in $\mathcal{L}$.

The elements of $\mathcal{L} / \mathcal{L}^{*}$ are the equivalence classes [A] obtained by adding to $\mathbf{A} \in \mathcal{L}$ an arbitrary matrix in $\mathcal{L}^{*}$,

$$
[\mathbf{A}]=\left\{\mathbf{A}+\mathbf{C}, \quad \mathbf{C} \in \mathcal{L}^{*}\right\}
$$

Every equivalence class [A] is an affine subspace of $\mathcal{L}$ and can also be represented as $[\mathbf{A}]=\mathbf{A}+\mathcal{L}^{*}$. Figure 1 illustrates an example of $[\mathbf{A}]$ in the case $n=3$.

We assume that every inconsistency index (5) assigns the same value, say 0 for simplicity, to every consistent matrix, see axiom 1 in (Brunelli and Fedrizzi 2015a) and Sect. 3.3.1. From what precedes, it is natural to assume that an inconsistency index assigns the same value to every matrix in a fixed equivalence class,

$$
\mathbf{A}, \mathbf{B} \in[\mathbf{A}] \Rightarrow I(\mathbf{B})=I(\mathbf{A})
$$

Now, in order to assign an inconsistency value $I(\cdot)$ to each equivalence class coherently with the assumption (6), the following must hold. For all $[\mathbf{A}] \in \mathcal{L} / \mathcal{L}^{*}$, every matrix $\mathbf{A} \in[\mathbf{A}]$ must have the same distance from $\mathcal{L}^{*}$. Corollary 1 will prove that this result can be achieved assuming, as in the following, that distance (6) is induced by a norm. 


\subsection{Inconsistency index as a seminorm}

Let $\|\mathbf{A}\|$ be a norm in $\mathbb{R}^{n \times n}$ and let

$$
d(\mathbf{A}, \mathbf{B})=\|\mathbf{A}-\mathbf{B}\|
$$

be the corresponding distance between matrices $\mathbf{A}$ and $\mathbf{B}$.

It is now possible to give our main definition.

Definition 4 Given a skew-symmetric matrix $\mathbf{A} \in \mathcal{L}$ and a norm $\|\cdot\|$ in $\mathbb{R}^{n \times n}$, the inconsistency index of $\mathbf{A}$ based on distance (11) is

$$
I_{d}(\mathbf{A})=d\left(\mathbf{A}, \mathcal{L}^{*}\right)=\min _{\mathbf{B} \in \mathcal{L}^{*}} d(\mathbf{A}, \mathbf{B})=\min _{\mathbf{B} \in \mathcal{L}^{*}}\|\mathbf{A}-\mathbf{B}\|=\left\|\mathbf{A}-\mathbf{A}^{*}\right\|
$$

where $\mathbf{A}^{*} \in \mathcal{L}^{*}$ is a solution of the minimization problem in (12).

Note that, by choosing the 1-norm $\|\cdot\|_{1}$, the optimization problem (12) is equivalent to the optimization problem (24) in (Brunelli and Cavallo 2020), and the same holds for the inconsistency index proposed there, except for a numerical constant. Most of the results in this paper are based on the following theorem.

Theorem 1 An inconsistency index $I_{d}(\mathbf{A})$ defined as a norm-based distance (12) is a seminorm in $\mathcal{L}$.

Proof It must be proved that

1. $I_{d}(k \mathbf{A})=|k| I_{d}(\mathbf{A}) \quad \forall \mathbf{A} \in \mathcal{L}, \quad \forall k \in \mathbb{R}$

2. $I_{d}\left(\mathbf{A}+\mathbf{A}^{\prime}\right) \leq I_{d}(\mathbf{A})+I_{d}\left(\mathbf{A}^{\prime}\right) \quad \forall \mathbf{A}, \mathbf{A}^{\prime} \in \mathcal{L}$.

Let $\mathbf{A}^{*} \in \mathcal{L}^{*}$ be a $d$-nearest consistent matrix to $\mathbf{A} \in \mathcal{L}$, as in (12). Then, it is possible to prove that $k \mathbf{A}^{*}$ is a $d$-nearest consistent matrix to $k \mathbf{A}$. Equality 1 . is obviously true for $k=0$. Then, let us assume $k \neq 0$. It is

$$
\left\|k \mathbf{A}-k \mathbf{A}^{*}\right\|=|k|\left\|\mathbf{A}-\mathbf{A}^{*}\right\| \leq|k|\|\mathbf{A}-\mathbf{B}\| \quad \forall \mathbf{B} \in \mathcal{L}^{*} .
$$

Since

$$
|k|\|\mathbf{A}-\mathbf{B}\|=\|k \mathbf{A}-k \mathbf{B}\|,
$$

then it is

$$
\left\|k \mathbf{A}-k \mathbf{A}^{*}\right\| \leq\|k \mathbf{A}-k \mathbf{B}\| \quad \forall \mathbf{B} \in \mathcal{L}^{*}
$$

It follows, from (13), that

$$
\left\|k \mathbf{A}-k \mathbf{A}^{*}\right\|=\min _{k \mathbf{B} \in \mathcal{L}^{*}}\|k \mathbf{A}-k \mathbf{B}\|=I_{d}(k \mathbf{A})
$$


The last equality follows from the fact that, for a fixed $k \neq 0$, every matrix of $\mathcal{L}^{*}$ can be expressed as $k \mathbf{B}$ with $\mathbf{B} \in \mathcal{L}^{*}$. Then,

$$
I_{d}(k \mathbf{A})=d\left(k \mathbf{A}, \mathcal{L}^{*}\right)=|k| d\left(\mathbf{A}, \mathcal{L}^{*}\right)=|k| I_{d}(\mathbf{A}) \quad \forall k \in \mathbb{R},
$$

so that the first property is proved. The second property can be proved as follows,

$$
\begin{aligned}
I_{d}\left(\mathbf{A}+\mathbf{A}^{\prime}\right) & =\min _{\mathbf{B} \in \mathcal{L}^{*}}\left\|\left(\mathbf{A}+\mathbf{A}^{\prime}\right)-\mathbf{B}\right\|= \\
& =\min _{\mathbf{C}, \mathbf{C}^{\prime} \in \mathcal{L}^{*}}\left\|\left(\mathbf{A}+\mathbf{A}^{\prime}\right)-\left(\mathbf{C}+\mathbf{C}^{\prime}\right)\right\| \\
& =\min _{\mathbf{C}, \mathbf{C}^{\prime} \in \mathcal{L}^{*}}\left\|(\mathbf{A}-\mathbf{C})+\left(\mathbf{A}^{\prime}-\mathbf{C}^{\prime}\right)\right\| \\
& \leq \min _{\mathbf{C} \in \mathcal{L}^{*}}\|(\mathbf{A}-\mathbf{C})\|+\min _{\mathbf{C}^{\prime} \in \mathcal{L}^{*}}\left\|\left(\mathbf{A}^{\prime}-\mathbf{C}^{\prime}\right)\right\| \\
& =I_{d}(\mathbf{A})+I_{d}\left(\mathbf{A}^{\prime}\right)
\end{aligned}
$$

where $\mathbf{B}=\mathbf{C}+\mathbf{C}^{\prime}$ and the arbitrariness of $\mathbf{C}$ and $\mathbf{C}^{\prime}$ in $\mathcal{L}^{*}$ follows from the one of $\mathbf{B}$.

Clearly, an inconsistency index $I_{d}(\mathbf{A})$ is not a norm in $\mathcal{L}$, since $I_{d}(\mathbf{A})=0 \forall \mathbf{A} \in \mathcal{L}^{*}$.

Theorem 2 If $\mathbf{A} \in \mathcal{L}$,

1. Every inconsistency index $I_{d} \mathbf{( A )}$ given by (12) is invariant with respect to addition of a consistent skew-symmetric matrix,

$$
I_{d}(\mathbf{A})=I_{d}(\mathbf{A}+\mathbf{B}) \quad \forall \mathbf{B} \in \mathcal{L}^{*}
$$

2. If $\mathbf{A}^{*} \in \mathcal{L}^{*}$ is a d-nearest consistent matrix to $\mathbf{A}$ and $\mathbf{B} \in \mathcal{L}^{*}$, then $\mathbf{A}^{*}+\mathbf{B}$ is a $d$-nearest consistent matrix to $\mathbf{A}+\mathbf{B}$

Proof For all $\mathbf{A} \in \mathcal{L}$ and $\mathbf{B} \in \mathcal{L}^{*}$, it is $I_{d}(\mathbf{B})=0$ and therefore

$$
I_{d}(\mathbf{A}+\mathbf{B}) \leq I_{d}(\mathbf{A})+I_{d}(\mathbf{B})=I_{d}(\mathbf{A})
$$

but also

$$
I_{d}(\mathbf{A})=I_{d}((\mathbf{A}+\mathbf{B})-\mathbf{B}) \leq I_{d}(\mathbf{A}+\mathbf{B})+I_{d}(-\mathbf{B})=I_{d}(\mathbf{A}+\mathbf{B}),
$$

since $\mathbf{B} \in \mathcal{L}^{*} \Rightarrow-\mathbf{B} \in \mathcal{L}^{*}$. Equality (17) follows from (18) and (19).

To prove the second statement, let us fix $\mathbf{B} \in \mathcal{L}^{*}$ and evaluate $\left\|(\mathbf{A}+\mathbf{B})-\left(\mathbf{A}^{*}+\mathbf{B}\right)\right\|$

$$
\begin{aligned}
\left\|(\mathbf{A}+\mathbf{B})-\left(\mathbf{A}^{*}+\mathbf{B}\right)\right\| & =\left\|\mathbf{A}-\mathbf{A}^{*}\right\| \\
& \leq\|\mathbf{A}-\mathbf{C}\| \quad \forall \mathbf{C} \in \mathcal{L}^{*}
\end{aligned}
$$




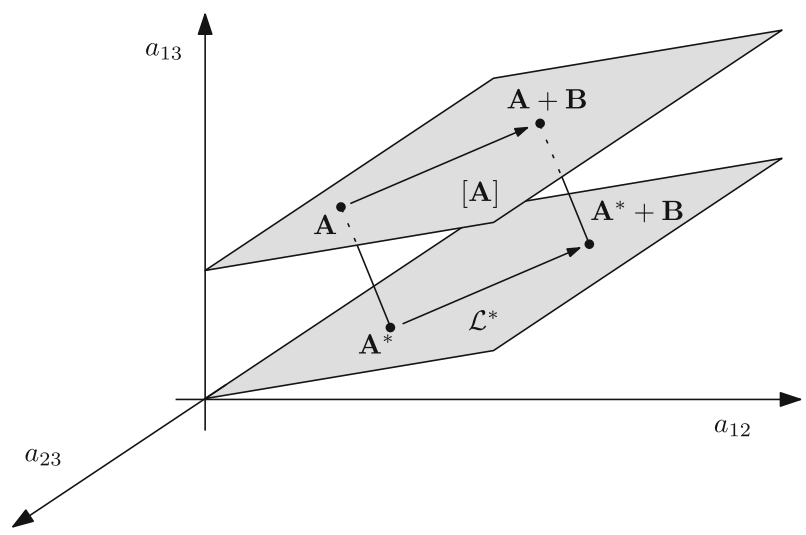

Fig. 2 Representation of Theorem 2 in the case $n=3$

Since

$$
\|\mathbf{A}-\mathbf{C}\|=\|(\mathbf{A}+\mathbf{B})-(\mathbf{C}+\mathbf{B})\|,
$$

then it is

$$
\left\|(\mathbf{A}+\mathbf{B})-\left(\mathbf{A}^{*}+\mathbf{B}\right)\right\| \leq\|(\mathbf{A}+\mathbf{B})-(\mathbf{C}+\mathbf{B})\| \quad \forall \mathbf{C} \in \mathcal{L}^{*}
$$

Figure 2 illustrates Theorem 2 in the case $n=3$.

Crawford (1987) introduced the so-called Geometric Consistency Index, GCI, and proved a theorem (Theorem 2 in the cited paper) which states, for his index, the same result as in Theorem 2 of this paper. He used the multiplicative representation of preferences, so that he proved the invariance with respect to the Hadamard product $\mathbf{A} \cdot \mathbf{B}=\left(a_{i j} b_{i j}\right)_{n \times n}$ instead of invariance with respect to the sum as in (17). Theorem 2 can therefore be viewed as an extension of Crawford's theorem to the general case of norm-based consistency indices in the additive representation of preferences. The semantic of Theorem 2 is as follows. The inconsistency degree $I_{d}(\mathbf{A})$ of a $P C M \mathbf{A}$ remains unchanged by adding consistent preferences.

From Theorem 2, the following corollary can be stated.

Corollary 1 If matrices $\mathbf{A}, \mathbf{B} \in \mathcal{L}$ are in the same equivalence class, then they have the same inconsistency index (12),

$$
\mathbf{A} \sim \mathbf{B} \Rightarrow I_{d}(\mathbf{A})=I_{d}(\mathbf{B})
$$

where $\sim$ is defined in (7).

The proof of (22) directly follows from (17) and (9).

Since $\mathcal{L}^{*}$ is the subspace of $\mathcal{L}$ where the seminorm $I_{d}(\cdot)$ is null, then this seminorm induces a norm in $\mathcal{L} / \mathcal{L}^{*}$. The result is described in the following corollary. 
Corollary 2 The function $I_{d}: \mathcal{L} / \mathcal{L}^{*} \rightarrow \mathbb{R}$, defined as follows

$$
I_{d}([\mathbf{A}])=I_{d}(\mathbf{A}), \quad \mathbf{A} \in[\mathbf{A}],
$$

is a norm on $\mathcal{L} / \mathcal{L}^{*}$.

Note that, for sake of simplicity, we use the same notation $I_{d}(\cdot)$ for the inconsistency index defined in $\mathcal{L}$, where $I_{d}$ is a seminorm, and the one defined in $\mathcal{L} / \mathcal{L}^{*}$, where $I_{d}$ is a norm.

If the norm in (11) arises from an inner product, $\|\mathbf{A}\|=\sqrt{\langle\mathbf{A}, \mathbf{A}\rangle}$, then it is possible to define the orthogonal complement $\left(\mathcal{L}^{*}\right)^{\perp}$ of $\mathcal{L}^{*}$, where $\left(\mathcal{L}^{*}\right)^{\perp}=\left\{\mathbf{A} \in \mathcal{L} \mid \mathbf{A} \perp \mathcal{L}^{*}\right\}$. Therefore, quotient space $\mathcal{L} / \mathcal{L}^{*}$ is isomorphic to $\left(\mathcal{L}^{*}\right)^{\perp}$ and $\mathcal{L}$ can be obtained as direct sum of $\mathcal{L}^{*}$ and $\left(\mathcal{L}^{*}\right)^{\perp}$,

$$
\mathcal{L}=\mathcal{L}^{*} \oplus\left(\mathcal{L}^{*}\right)^{\perp}
$$

Property (24) extends the result of Barzilai (1998), where he proposed the orthogonal decomposition of a matrix $\mathbf{A} \in \mathcal{L}$ into its 'consistent' and 'totally inconsistent' components, $\mathbf{A}=\mathbf{C}+\mathbf{E}$, and orthogonality $\mathbf{C} \perp \mathbf{E}$ refers to the standard dot product on $\mathbb{R}^{n \times n}$ corresponding to the Euclidean norm. Note that the decomposition proposed by Barzilai has been further investigated and generalized by Cavallo (2019).

\subsection{Axioms satisfaction}

Brunelli and Fedrizzi (2015a) proposed five characterizing properties, or axioms, for an inconsistency index. Then, Brunelli (2017) completed the set of axioms with a sixth one. The axioms can be formulated both in the additive and in the multiplicative representation of preferences. We clearly refer to the former. In this section, we prove that an inconsistency index $I_{d}(\mathbf{A})$, given by (12), satisfies all the six axioms, provided that (11) is a permutation-invariant norm on $\mathbb{R}^{n \times n}$, that is a norm invariant with respect to permutations on the coordinates.

\subsubsection{Axiom (A1): Existence of a unique element representing consistency}

The first characterizing property (A1) requires that an inconsistency index must be associated to a unique real number which represents perfectly consistent preferences. More formally, it is demanded that

$$
\exists ! v \in \mathbb{R} \text { such that } I(\mathbf{A})=v \Leftrightarrow \mathbf{A} \in \mathcal{L}^{*} .
$$

An inconsistency index (12) satisfies axiom (A1) with $v=0$, since $d\left(\mathbf{A}, \mathcal{L}^{*}\right)=0 \Leftrightarrow$ $\mathbf{A} \in \mathcal{L}^{*}$.

\subsubsection{Axiom (A2): Invariance under permutation of alternatives}

Axiom (A2) requires that an inconsistency index must be independent from the order of the alternatives, i.e., from the order to which the alternatives are associated with the 
rows and columns of $\mathbf{A}$,

$$
I\left(\mathbf{P A} \mathbf{P}^{T}\right)=I(\mathbf{A}) \quad \forall \mathbf{A} \in \mathcal{L},
$$

for any permutation matrix $\mathbf{P}$.

If the norm in (12) is permutation invariant, then axiom (A2) is satisfied.

\subsubsection{Axiom (A3): Monotonicity under reciprocity-preserving mapping}

We first briefly summarize axiom (A3). For a more comprehensive description, please refer to Brunelli and Fedrizzi (2015a). The idea underlying axiom (A3) is the following: if inconsistent preferences are intensified, then a better value of an inconsistency index cannot be obtained. By 'preference intensification,' we mean going farther from complete indifference $a_{i j}=0 \forall i, j$, which is clearly fully consistent. Going farther from this, uniformity means having stronger judgments and this should not make their possible inconsistency less evident. It can be proved that the only transformation $a_{i j} \rightarrow f\left(a_{i j}\right)$ which can intensify preferences by preserving the necessary additive reciprocity structure $(3)$ is

$$
f\left(a_{i j}\right)=k a_{i j}, \quad k>1
$$

or, equivalently,

$$
\mathbf{A} \rightarrow k \mathbf{A}, \quad k>1
$$

The formalization of Axiom (A3) is as follows.

Axiom (A3) An inconsistency index $I(\cdot)$ satisfies axiom (A3) if and only if

$$
I(k \mathbf{A}) \geq I(\mathbf{A}) \quad \forall k>1 \quad \forall \mathbf{A} \in \mathcal{L}
$$

Proposition 1 An inconsistency index defined by (12) satisfies axiom (A3).

Proof Theorem 1 implies that $I_{d}(k \mathbf{A})=|k| I_{d}(\mathbf{A}) \quad \forall k \in \mathbb{R}$. Then, $I_{d}(k \mathbf{A})>$ $I_{d}$ (A) $\forall k>1$ and axiom (A3) is satisfied.

\subsubsection{Axiom (A4): Monotonicity on single entries}

We briefly summarize axiom (A4). The reader can refer to Brunelli and Fedrizzi (2015a) for a more comprehensive description. The idea underlying axiom (A4) is that an inconsistency index is non-decreasing with respect to an elementary modification of a consistent matrix. More formally, given a consistent skew-symmetric matrix $\mathbf{A} \in \mathcal{L}^{*}$ and $b \in \mathbb{R}, \quad b \neq 0$, let $\mathbf{A}_{p q}(b) \in \mathcal{L}$ be the inconsistent skew-symmetric matrix obtained from $\mathbf{A}$ by replacing the single entry $a_{p q}$ with $a_{p q}+b, p, q \in\{1, \ldots, n\}$, $p \neq q$. Clearly, $a_{q p}$ must be replaced with $a_{q p}-b$ in order to preserve skew-symmetry. Axiom (A4) requires that the larger the change of $a_{p q}$ from its consistent value, the 
more inconsistent the obtained matrix is. The formalization of Axiom (A4) is as follows.

Axiom (A4) An inconsistency index $I(\cdot)$ satisfies axiom (A4) if and only if $I\left(\mathbf{A}_{p q}(b)\right)$ is a non-decreasing function of $b$ for $b>0$ and a non-increasing function of $b$ for $b<0, \forall \mathbf{A} \in \mathcal{L}^{*}, \forall p, q \in\{1, \ldots, n\}, p \neq q$.

Proposition 2 An inconsistency index defined by (12) satisfies axiom (A4).

Proof The proof follows from the homogeneity of seminorms. Given a consistent skew-symmetric matrix $\mathbf{A} \in \mathcal{L}^{*}$ and $b \in \mathbb{R}, b \neq 0$, let $\mathbf{A}_{p q}(b) \in \mathcal{L}$ be the inconsistent skew-symmetric matrix obtained as described above. Matrix $\mathbf{A}_{p q}(b)$ can be obtained as follows,

$$
\mathbf{A}_{p q}(b)=\mathbf{A}+b \mathbf{M}_{p q}
$$

where $\mathbf{M}_{p q}$ is the $n \times n$ matrix with all null entries except $\mathbf{M}_{p q}(p, q)=1$, $\mathbf{M}_{p q}(q, p)=-1$. Theorem 2 implies that all the inconsistency of matrix $\mathbf{A}_{p q}(b)$ is due to matrix $b \mathbf{M}_{p q}$, when inconsistency is evaluated by means of $I_{d}(\cdot)$,

$$
I_{d}\left(\mathbf{A}_{p q}(b)\right)=I_{d}\left(\mathbf{A}+b \mathbf{M}_{p q}\right)=I_{d}\left(b \mathbf{M}_{p q}\right)
$$

Theorem 1 implies that $I_{d}\left(b \mathbf{M}_{p q}\right)=|b| I_{d}\left(\mathbf{M}_{p q}\right) \quad \forall b \in \mathbb{R}$. Since $I_{d}\left(\mathbf{M}_{p q}\right)>0$, then axiom (A4) is satisfied.

\subsubsection{Axiom (A5): Continuity}

Axiom (A5) requires continuity of an inconsistency index. Continuity of (12) directly follows from the continuity of norms.

\subsubsection{Axiom (A6): Invariance under inversion of preferences}

Axiom (A6) (Brunelli 2017) requires that an inconsistency index does not change when preferences expressed in the form of a pairwise comparison matrix $\mathbf{A}$ are inverted by taking its transpose $\mathbf{A}^{T}$. The idea underlying axiom (A6) is that by inverting all the preferences we change their polarity, but leave their structure unchanged. The formalization of Axiom (A6) is as follows. Axiom (A6) An inconsistency index $I(\cdot)$ satisfies axiom (A6) if and only if $I(\mathbf{A})=I\left(\mathbf{A}^{T}\right) \quad \forall \mathbf{A} \in \mathcal{L}$.

Proposition 3 An inconsistency index defined by (12) satisfies axiom (A6).

Proof Since $\mathbf{A}$ is skew-symmetric, it is $\mathbf{A}^{T}=-\mathbf{A}$. Then, an inconsistency index $I(\cdot)$ satisfies axiom (A6) if and only if $I(\mathbf{A})=I\left(\mathbf{A}^{T}\right)=I(-\mathbf{A}) \quad \forall \mathbf{A} \in \mathcal{L}$. By defining $\mathbf{B}^{\prime}:=-\mathbf{B}$, it is

$$
I_{d}(-\mathbf{A})=\min _{\mathbf{B} \in \mathcal{L}^{*}} d(-\mathbf{A}, \mathbf{B})=\min _{\mathbf{B} \in \mathcal{L}^{*}}\|-\mathbf{A}-\mathbf{B}\|
$$




$$
\begin{aligned}
& =\min _{\mathbf{B}^{\prime} \in \mathcal{L}^{*}}\left\|-\mathbf{A}+\mathbf{B}^{\prime}\right\|=\min _{\mathbf{B}^{\prime} \in \mathcal{L}^{*}}|-1|\left\|\mathbf{A}-\mathbf{B}^{\prime}\right\| \\
& =\min _{\mathbf{B}^{\prime} \in \mathcal{L}^{*}}\left\|\mathbf{A}-\mathbf{B}^{\prime}\right\|=I_{d}(\mathbf{A}),
\end{aligned}
$$

Similarly to (14), the proof follows from the fact that every matrix $\mathbf{B}$ of $\mathcal{L}^{*}$ can be expressed as $\mathbf{B}=-\mathbf{B}^{\prime}$ with $\mathbf{B}^{\prime} \in \mathcal{L}^{*}$. Then, axiom (A6) is satisfied by (12).

\subsection{Boundary property for group decision making}

In this section, we prove that inconsistency index $I_{d}(\mathbf{A})$ given by (12) satisfies the upper bound property described by Brunelli and Fedrizzi (2015b).

If the preferences of $k=1, \ldots, m$ decision makers are expressed by means of $m$ $P C M \mathrm{~s} \mathbf{A}^{k}=\left(a_{i j}^{k}\right)$, it is a relevant problem to study the group $P C M \mathbf{A}^{G}=\left(a_{i j}^{G}\right)$ obtained by aggregating the $m$ individual $P C M$ s. Dijkstra (2012) proved that, if the $m$ $P C M$ s are expressed in the multiplicative representation of preferences, the weighted geometric mean is the unique aggregation method that guarantees some important properties of the group PCM to hold. Let us consider

$$
a_{i j}^{G}=\prod_{k=1}^{m}\left(a_{i j}^{k}\right)^{\lambda_{k}},
$$

where $\left(\lambda_{1}, \ldots, \lambda_{m}\right)$ is the weight vector of the decision makers such that $\lambda_{k} \geq 0$, $\sum_{k=1}^{m} \lambda_{k}=1$ and $\mathbf{A}^{k}=\left(a_{i j}^{k}\right) \in \mathcal{A}$. In particular, (32) is the unique consistencypreserving non-trivial aggregation method. Brunelli and Fedrizzi (2015b) studied how the inconsistency of $\mathbf{A}^{G}$ depends on the inconsistency of $\mathbf{A}^{k}, k=1, \ldots, m$. They proved, in particular, that some known inconsistency indices satisfy the following upper-bound property.

Definition 5 (Brunelli and Fedrizzi 2015b) A function $I: \mathcal{A} \rightarrow \mathbb{R}$ is upper bounded w.r.t the geometric mean if:

$$
I\left(\mathbf{A}^{G}\right) \leq \max \left\{I\left(\mathbf{A}^{1}\right), \ldots, I\left(\mathbf{A}^{m}\right)\right\} .
$$

The following property is more restrictive than (33), it was also introduced by Brunelli and Fedrizzi (2015b) and studied for some known inconsistency indices.

Definition 6 (Brunelli and Fedrizzi 2015b) A function $I: \mathcal{A} \rightarrow \mathbb{R}$ is strongly upper bounded w.r.t the geometric mean if:

$$
I\left(\mathbf{A}^{G}\right) \leq \sum_{k=1}^{m} \lambda_{k} I\left(\mathbf{A}^{k}\right),
$$

where $\left(\lambda_{1}, \ldots, \lambda_{m}\right)$ is the same weight vector used in (32) to obtain $\mathbf{A}^{G}$.

Let us study the upper-bound properties (33) and (34) from the additive approach described above, that is, in the vector space $\mathcal{L}$ of skew-symmetric preference matrices. 
By componentwise applying the logarithmic function (2) as described in Section 2, the aggregation (32) in $\mathcal{A}$ clearly corresponds to a linear combination in $\mathcal{L}$. By using, for simplicity, the same notation as in (32), the group skew-symmetric preference matrix is given by

$$
\begin{aligned}
& a_{i j}^{G}=\sum_{k=1}^{m} \lambda_{k}\left(a_{i j}^{k}\right) \quad i, j=1, \ldots, n \\
& \mathbf{A}^{G}=\sum_{k=1}^{m} \lambda_{k} \mathbf{A}^{k}
\end{aligned}
$$

where $\mathbf{A}^{k}=\left(a_{i j}^{k}\right) \in \mathcal{L}$. The following result holds.

Theorem 3 An inconsistency index $I_{d}(\mathbf{A})$ defined as a norm-based distance (12) is strongly upper bounded, i.e., it satisfies the upper boundary property (34).

Proof Theorem 1 implies that

$$
\begin{aligned}
I_{d}\left(\mathbf{A}^{G}\right) & =I_{d}\left(\sum_{k=1}^{m} \lambda_{k} \mathbf{A}^{k}\right) \leq \sum_{k=1}^{m} I_{d}\left(\lambda_{k} \mathbf{A}^{k}\right) \\
& =\sum_{k=1}^{m}\left|\lambda_{k}\right| I_{d}\left(\mathbf{A}^{k}\right)=\sum_{k=1}^{m} \lambda_{k} I_{d}\left(\mathbf{A}^{k}\right)
\end{aligned}
$$

\section{Conclusions and future work}

In this paper, we showed that by defining an inconsistency index $I_{d}(\mathbf{A})$ by means of a distance induced by a norm, as in (12), it is possible to prove many relevant properties of this index. Note that the property of homogeneity of a (semi)norm is a crucial assumption in proving several Theorems and Propositions. Nevertheless, a norm is, in our view, a sufficiently general notion to satisfy different requirements of a decision maker. Let us clarify our point with the example of the $p$-norm of a vector $\mathbf{x}=\left(x_{1}, \ldots, x_{n}\right)$,

$$
\|\mathbf{x}\|_{p}=\left(\sum_{i=1}^{n}\left|x_{i}\right|^{p}\right)^{\frac{1}{p}}, \quad p \geq 1
$$

A $p$-norm is clearly a permutation-invariant norm, so that all the results in Sect. 3 hold if $I_{d}(\mathbf{A})$ is defined by means of a $p$-norm (38). Note that the well-known Geometric Consistency Index GCI introduced by Crawford (1987) corresponds, in the additive representation of preferences, to the usual Euclidean norm $\|\cdot\|_{2}$. More generally, if a decision maker uses $p$-norm (38), he/she is provided with a flexible tool in emphasizing 
inconsistent preferences. More precisely, the larger $p$, the more emphasis is paid in order to avoid large differences between the elicited preferences and the closest consistent ones.

In future research, we will study the relationship between the 'natural' inconsistency ranking defined by Csató (2019) and the ranking on the set of triads induced by the norm-based inconsistency indices defined in this paper. More precisely, we will study how the choice of a norm affects this relationship. A second research topic will be the possible relationship between our inconsistency index and the index introduced by Koczkodaj (1993).

Funding Open access funding provided by Universitá degli Studi di Trento within the CRUI-CARE Agreement.

Open Access This article is licensed under a Creative Commons Attribution 4.0 International License, which permits use, sharing, adaptation, distribution and reproduction in any medium or format, as long as you give appropriate credit to the original author(s) and the source, provide a link to the Creative Commons licence, and indicate if changes were made. The images or other third party material in this article are included in the article's Creative Commons licence, unless indicated otherwise in a credit line to the material. If material is not included in the article's Creative Commons licence and your intended use is not permitted by statutory regulation or exceeds the permitted use, you will need to obtain permission directly from the copyright holder. To view a copy of this licence, visit http://creativecommons.org/licenses/by/4.0/.

\section{References}

Barzilai, J.: Consistency measures for pairwise comparison matrices. J. Multi-Criteria Decis. Anal. 7(3), 123-132 (1998)

Bozóki, S., Rapcsák, T.: On Saaty’s and Koczkodaj's inconsistencies of pairwise comparison matrices. J. Glob. Optim. 42(2), 157-175 (2008)

Brunelli, M.: Studying a set of properties of inconsistency indices for pairwise comparisons. Ann. Oper. Res. 248(1-2), 143-161 (2017)

Brunelli, M.: A survey of inconsistency indices for pairwise comparisons. Int. J. Gen. Syst. 47(8), 751-771 (2018)

Brunelli, M., Cavallo, B.: Distance-based measures of incoherence for pairwise comparisons. Knowl. Based System. 187, 104808 (2020)

Brunelli, M., Fedrizzi, M.: Axiomatic properties of inconsistency indices for pairwise comparisons. J. Oper. Res. Soc. 61(1), 1-15 (2015a)

Brunelli, M., Fedrizzi, M.: Boundary properties of the inconsistency of pairwise comparisons in group decisions. Eur. J. Oper. Res. 240(3), 765-773 (2015b)

Brunelli, M., Fedrizzi, M.: A general formulation for some inconsistency indices of pairwise comparisons. Ann. Oper. Res. 274(1-2), 155-169 (2019)

Brunelli, M., Canal, L., Fedrizzi, M.: Inconsistency indices for pairwise comparison matrices: a numerical study. Ann. Oper. Res. 211(1), 493-509 (2013)

Cavallo, B.: $\mathcal{G}$-distance and $\mathcal{G}$-decomposition for improving $\mathcal{G}$-consistency of a Pairwise Comparison Matrix. Fuzzy Optim. Decis. Mak. 18(1), 57-83 (2019)

Cavallo, B.: Functional relations and Spearman correlation between consistency indices. J. Oper. Res. Soc. 71(2), 301-311 (2020)

Cavallo, B., D'Apuzzo, L.: A general unified framework for pairwise comparison matrices in multicriterial methods. Int. J. Intell. Syst. 24(4), 377-398 (2009)

Chu, M.T.: On the optimal consistent approximation to pairwise comparison matrices. Linear Algebra Appl. 272, 155-168 (1998)

Crawford, G.: The geometric mean procedure for estimating the scale of a judgement matrix. Math. Model. 9(3-5), 327-334 (1987) 
Crawford, G., Williams, C.: A note on the analysis of subjective judgement matrices. J. Math. Psychol. 29, 25-40 (1985)

Csató, L.: Characterization of an inconsistency ranking for pairwise comparison matrices. Ann. Oper. Res. 261(1-2), 155-165 (2018)

Csató, L.: Axiomatizations of inconsistency indices for triads. Ann. Oper. Res. 280(1-2), 99-110 (2019)

Dijkstra, T.K.: On the extraction of weights from pairwise comparison matrices. Cent. Eur. J. Oper. Res. 21(1), 103-123 (2013)

Duszak, Z., Koczkodaj, W.W.: Generalization of a new definition of consistency for pairwise comparisons. Inf. Process. Lett. 52(5), 273-276 (1994)

Fedrizzi, M., Brunelli, M., Caprila, A.: The linear algebra of pairwise comparisons. Int. J. Approx. Reason. 118, 190-207 (2019)

Fichtner, J.: Some thoughts about the mathematics of the analytic hierarchy process. Report 8403, Institut für Angewandte Systemforschung und Operations Research, Hochschule der Bundeswehr München (1984)

Fichtner, J.: On deriving priority vectors from matrices of pairwise comparisons. Socio-Econ. Plan. Sci. 20, 341-345 (1986)

Koczkodaj, W.W.: A new definition of consistency of pairwise comparisons. Math. Comput. Model. 18(7), 79-84 (1993)

Koczkodaj, W.W., Orlowski, M.: An orthogonal basis for computing a consistent approximation to a pairwise comparison matrix. Comput. Math. Appl. 34(10), 41-47 (1997)

Mazurek, J., Ramík, J.: Some new properties of inconsistent pairwise comparisons matrices. Int. J. Approx. Reason. 113, 119-132 (2019)

Ramík, J., Korviny, P.: Inconsistency of pair-wise comparison matrix with fuzzy elements based on geometric mean. Fuzzy Sets Syst. 161(11), 1604-1613 (2010)

Saaty, T.L.: A scaling method for priorities in hierarchical structures. J. Math. Psychol. 15, 234-281 (1977)

Saaty, T.L.: Highlights and critical points in the theory and application of the Analytic Hierarchy Process. Eur. J. Oper. Res. 74, 426-447 (1994)

Publisher's Note Springer Nature remains neutral with regard to jurisdictional claims in published maps and institutional affiliations. 